\title{
Correction to: Cerebrovascular and amyloid pathology in predementia stages: the relationship with neurodegeneration and cognitive decline
}

Isabelle Bos ${ }^{1 *}$, Frans R. Verhey ${ }^{1}$, Inez H. G. B. Ramakers ${ }^{1}$, Heidi I. L. Jacobs ${ }^{1}$, Hilkka Soininen ${ }^{2,3}$, Yvonne Freund-Levi ${ }^{4}$, Harald Hampel ${ }^{5,6}$, Magda Tsolaki $^{7}$, Åsa K. Wallin ${ }^{8}$, Mark A. van Buchem ${ }^{9}$, Ania Oleksik ${ }^{10}$, Marcel M. Verbeek ${ }^{11}$, Marcel Olde Rikkert ${ }^{12}$, Wiesje M. van der Flier ${ }^{13}$, Philip Scheltens ${ }^{13}$, Pauline Aalten ${ }^{1}$, Pieter Jelle Visser ${ }^{1,13}$ and Stephanie J. B. Vos ${ }^{1 *}$

\section{Correction}

Upon publication of this article [1], it was noticed that there were some inconsistencies in Tables 1,2 and 3. Some of the superscript letters were incorrectly assigned. Please see below the correct tables:

\footnotetext{
Author details

'Department of Psychiatry and Neuropsychology, School of Mental Health and Neuroscience, Alzheimer Center Limburg, Maastricht University, Maastricht, The Netherlands. ${ }^{2}$ Institute of Clinical Medicine, Neurology, University of Eastern Finland, Kuopio, Finland. ${ }^{3}$ Neurocenter and Department of Neurology, Kuopio University Hospital, Kuopio, Finland. ${ }^{4}$ Department of Neurobiology, Caring Sciences and Society (NVS), Karolinska University Hospital Huddinge, Stockholm, Sweden. ${ }^{5}$ AXA Research Fund and UPMC Chair Sorbonne Universités, Université Pierre et Marie Curie (UPMC), Paris, France. ${ }^{6}$ Institut du cerveau et de la moelle (ICM), Hôpital Pitié-Salpêtrière, Paris, France. ${ }^{7}$ Memory and Dementia Center, 3rd Department of Neurology, Aristotle University of Thessaloniki, G Papanicolau" General Hospital, Thessaloniki, Greece. ${ }^{8}$ Department of Clinical Sciences Malmö, Clinical Memory Research Unit, Lund University, Lund, Sweden. ${ }^{9}$ Department of Radiology, Leiden University Medical Center, Leiden, The Netherlands.

${ }^{10}$ Department of Gerontology and Geriatrics, Leiden University Medical Center, Leiden, The Netherlands. ${ }^{11}$ Departments of Neurology and Laboratory Medicine, Donders Institute for Brain, Cognition and Behaviour, Radboud Alzheimer Center, Radboud University Medical Center, Nijmegen, The Netherlands. ${ }^{12}$ Radboudumc Alzheimer Centre, Department of Geriatric Medicine, Radboud University Medical Center, Nijmegen, The Netherlands.

${ }^{13}$ Department of Neurology, Alzheimer Centre, Neuroscience Campus Amsterdam, VU University Medical Center, Amsterdam, Netherlands.
}

Received: 20 February 2018 Accepted: 30 May 2018 Published online: 20 June 2018

\section{Reference}

1. Bos I, Verhey FR, Ramakers IHGB, Jacobs HIL, Soininen H, Freund-Levi Y, Hampel H, Tsolaki M, Wallin ÅK, van Buchem MA, Oleksik A, Verbeek MM, Olde Rikkert M, van der Flier WM, Scheltens P, Aalten P, Visser PJ, Vos SJB. Cerebrovascular and amyloid pathology in predementia stages: the relationship with neurodegeneration and cognitive decline. Alzheimers Res Ther. 2017;9:101. https://doi.org/10.1186/s13195-017-0328-9.

* Correspondence: isabelle.bos@maastrichtuniversity.nl

'Department of Psychiatry and Neuropsychology, School of Mental Health and Neuroscience, Alzheimer Center Limburg, Maastricht University, Maastricht, The Netherlands 
Table 1 Comparisons of baseline and follow-up characteristics by A 3 and $W M H$ status

\begin{tabular}{|c|c|c|c|c|}
\hline & $\begin{array}{l}\text { A } \beta-W M H- \\
n=140\end{array}$ & $\begin{array}{l}\mathrm{A} \beta-\mathrm{WMH}+ \\
n=39\end{array}$ & $\begin{array}{l}\mathrm{A} \beta+\mathrm{WMH}- \\
n=63\end{array}$ & $\begin{array}{l}\mathrm{A} \beta+\mathrm{WMH}+ \\
n=29\end{array}$ \\
\hline \multicolumn{5}{|l|}{ Baseline characteristics } \\
\hline Age & $61.7(8.3)^{B, C, D}$ & $71.3(7.7)^{\mathrm{A}, \mathrm{C}}$ & $66.7(7.8)^{\mathrm{A}, \mathrm{B}, \mathrm{D}}$ & $74.1(5.0)^{\mathrm{A}, \mathrm{C}}$ \\
\hline Female, $n$ & $94(67)^{C}$ & $23(59)$ & $32(51)^{\mathrm{A}}$ & $16(55)$ \\
\hline Education in years & $10.9(3.1)$ & $11.9(3.3)$ & $11.1(3.1)$ & $10.3(2.9)$ \\
\hline Hypertension, $\mathrm{n}^{*}$ & $43(34)$ & $9(25)$ & $15(25)$ & $9(32)$ \\
\hline Obesity, $\mathrm{n}^{*}$ & $15(14)$ & $3(11)$ & $4(8)$ & $4(21)$ \\
\hline Diabetes, $\mathrm{n}^{*}$ & $16(21)$ & $3(15)$ & $3(7)$ & $5(28)$ \\
\hline APOE- $\varepsilon 4$ carrier, $n^{*}$ & $33(51)^{B}$ & $5(24)^{A, C, D}$ & $29(62)^{B}$ & $10(56)^{B}$ \\
\hline Diagnosis $\mathrm{MCl}, \mathrm{n}$ & $70(50)^{\mathrm{D}}$ & $21(54)^{\mathrm{D}}$ & $40(64)$ & $22(76)^{A, B}$ \\
\hline amnestic $\mathrm{MCl}$ (\% within $\mathrm{MCl}$ group) & $40(57)$ & $15(71)$ & $27(68)$ & $17(77)$ \\
\hline non-amnestic $\mathrm{MCl}$ (\% within $\mathrm{MCl}$ group) & $30(43)$ & $6(29)$ & $13(33)$ & $5(23)$ \\
\hline CSF A $\beta 1-42, \mathrm{pg} / \mathrm{ml}$ & $973.6(312.0)^{C, D}$ & $885.0(242.0)^{C, D}$ & $404.3(102.6)^{A, B}$ & $419.3(97.2)^{A, B}$ \\
\hline White matter hyperintensities $^{\dagger}$ & $0.7(0.5)^{B, D}$ & $2.3(0.4)^{A, C}$ & $0.8(0.4)^{B, D}$ & $2.4(0.5)^{\mathrm{A}, \mathrm{C}}$ \\
\hline \multicolumn{5}{|l|}{ Follow-up characteristics } \\
\hline Follow-up time & $2.1(1.5)$ & $2.2(1.3)$ & $2.1(1.2)$ & $2.4(1.2)$ \\
\hline Time to progression to dementia & $1.3(0.5)^{B}$ & $2.0(0.7)^{\mathrm{A}}$ & $1.7(0.7)$ & $2.1(1.2)$ \\
\hline Progression to dementia, $\mathrm{n}$ & $8(6)^{B, C, D}$ & $9(23)^{A}$ & $18(29)^{\mathrm{A}}$ & $11(38)^{\mathrm{A}}$ \\
\hline - AD-type dementia, $n$ & $2(1)^{B, C, D}$ & $7(18)^{A}$ & $18(29)^{\mathrm{A}}$ & $10(35)^{\mathrm{A}}$ \\
\hline - Vascular dementia, n & $0(0)$ & $2(5)$ & $0(0)$ & $1(3)$ \\
\hline - Frontotemporal dementia, n & $4(3)$ & $0(0)$ & $0(0)$ & $0(0)$ \\
\hline - Lewy Body dementia, n & $1(1)$ & $0(0)$ & $0(0)$ & $0(0)$ \\
\hline - Dementia with unknown etiology, n & $1(1)$ & $0(0)$ & $0(0)$ & $0(0)$ \\
\hline
\end{tabular}

Results are mean (SD) for continuous variables or frequency (\%). Hypertension, obesity, diabetes and APOE ع4 genotype were only available in a subgroup of the sample Abbreviations: $A \beta$ amyloid-beta, $A D$ Alzheimer's disease, $A P O E$ Apolipoprotein $\mathrm{E}, \mathrm{MCl}$ mild cognitive impairment

${ }^{\dagger}$ WMH measured by the Fazekas scale, range $0-3$

${ }^{A} p<0.05$ compared to $A \beta-W M H-$

${ }^{\mathrm{B}} p<0.05$ compared to $\mathrm{A} \beta-\mathrm{WMH}+$

$C_{p}<0.05$ compared to $A \beta+W M H-$

$\mathrm{D}_{p}<0.05$ compared to $\mathrm{A} \beta+\mathrm{WMH}+$

Table 2 Values of neurodegenerative markers by A $/$ WMH groups

\begin{tabular}{lllll}
\hline & $\mathrm{A} \beta$ - WMH- & $\mathrm{A} \beta-\mathrm{WMH}+$ & $\mathrm{A} \beta+\mathrm{WMH}-$ & $\mathrm{A} \beta+\mathrm{WMH}+$ \\
\hline Neurodegeneration markers & $\mathrm{n}=140$ & $\mathrm{n}=39$ & $\mathrm{n}=63$ & $\mathrm{n}=29$ \\
MTA score & $1.2(1.2)^{\mathrm{B}, \mathrm{C}, \mathrm{D}}$ & $2.6(1.6)^{\mathrm{A}, \mathrm{D}}$ & $2.1(1.6)^{\mathrm{A}, \mathrm{D}}$ & $3.4(1.8)^{\mathrm{A}, \mathrm{B}, \mathrm{C}}$ \\
$\quad$ MTA abnormal, n & $62(45)^{\mathrm{B}, \mathrm{C}, \mathrm{D}}$ & $32(82)^{\mathrm{A}}$ & $41(67)^{\mathrm{A}, \mathrm{D}}$ & $26(93)^{\mathrm{A}, \mathrm{C}}$ \\
P-tau, pg/ml & $54.5(27.7)^{\mathrm{C}}$ & $63.2(29.3)$ & $77.0(56.3)^{\mathrm{A}}$ & $65.2(38.2)$ \\
$\quad$ P-tau abnormal, n & $53(38)^{\mathrm{C}}$ & $22(58)$ & $45(71)^{\mathrm{A}}$ & $15(52)$ \\
T-tau, pg/ml & $314.7(202.0)^{\mathrm{B}, \mathrm{C}, \mathrm{D}}$ & $438.4(248.0)^{\mathrm{A}}$ & $499.3(413.8)^{\mathrm{A}}$ & $426.2(275.2)^{\mathrm{A}}$ \\
$\quad$ T-tau abnormal, n & $36(26)^{\mathrm{B}, \mathrm{C}, \mathrm{D}}$ & $20(53)^{\mathrm{A}}$ & $36(57)^{\mathrm{A}}$ & $14(48)^{\mathrm{A}}$ \\
\hline
\end{tabular}

Results are mean (SD) and number (\%). All analyses were adjusted for study, baseline diagnosis and demographics

Abbreviations: $A \beta$ amyloid-beta, MTA medial temporal lobe atrophy, P-tau phosphorylated tau, T-tau Total tau, WMH white matter hyperintensities

${ }^{\mathrm{A}} p<0.05$ compared to $A \beta-W M H-$

${ }^{\mathrm{B}} p<0.05$ compared to $\mathrm{A} \beta-\mathrm{WMH}+$.

$C_{p}<0.05$ compared to $A \beta+W M H-$.

$\mathrm{D}_{\mathrm{p}}<0.05$ compared to $A \beta+\mathrm{WMH}+$. 
Table 3 Cognitive performance and decline by $A \beta / W M H$ groups

\begin{tabular}{llllll}
\hline & & $A \beta-W M H-$ & $A \beta-W M H+$ & $A \beta+W M H-$ & $A \beta+W M H+$ \\
\hline MMSE & $n$ & 140 & 39 & 62 & 27 \\
& Baseline & $27.79(27.39,28.19)$ & $27.52(26.83,28.21)$ & $27.20(26.62,27.78)$ & $27.40(26.54,28.25)$ \\
& Slope & $-0.01(-0.15,0.12)$ & $-0.29(-0.55,-0.02)$ & $-0.22(-0.44,-0.01)$ & $-0.31(-0.62,0.00)$ \\
Memory delayed recall z-score & $\mathrm{n}$ & 133 & 37 & 58 & 27 \\
& Baseline & $-0.48(-0.72,-0.24)^{\mathrm{B}, \mathrm{C}, \mathrm{D}}$ & $-1.04(-1.48,-0.61)^{\mathrm{A}}$ & $-1.04(-1.41,-0.68)^{\mathrm{A}}$ & $-1.33(-1.86,-0.80)^{\mathrm{A}}$ \\
& Slope & $0.05(-0.03,0.13)$ & $0.02(-0.12,0.17)$ & $0.02(-0.11,0.14)$ & $-0.07(-0.24,0.09)$ \\
Executive functioning z-score & $\mathrm{n}$ & 130 & 37 & 60 & 24 \\
& Baseline & $-0.48(-0.76,-0.21)$ & $-0.41(-0.92,0.09)$ & $-0.78(-1.18,-0.37)$ & $-1.12(-1.73,-0.50)$ \\
& Slope & $0.06(-0.02,0.13)$ & $-0.00(-0.15,0.15)$ & $-0.03(-0.16,0.10)$ & $-0.04(-0.23,0.15)$
\end{tabular}

Results are mean (95\% confidence interval). Bold slope estimates $=p<0.05$. All analyses were adjusted for study. The analyses on MMSE scores were also corrected for demographics and baseline diagnosis

Abbreviations: $A \beta$ amyloid-beta, MMSE mini mental state examination, $W M H$ white matter Hyperintensities

$\mathrm{A}_{\mathrm{p}}<0.05$ compared to $A \beta-\mathrm{WMH}-$

${ }^{B} \mathrm{p}<0.05$ compared to $A \beta-W M H+$.

$c_{p}<0.05$ compared to $A \beta+W M H-$.

$\mathrm{D}_{\mathrm{p}}<0.05$ compared to $A \beta+\mathrm{WMH}+$. 